\title{
Towards the closure of Collar's triangle by optical diagnostics
}

\author{
G. González Saiz ${ }^{1 *}$, A. Sciacchitano ${ }^{1}$, F. Scarano ${ }^{1}$ \\ Faculty of Aerospace Engineering, Delft University of Technology, Delft, The Netherlands \\ *g.gonzalezsaiz@tudelft.nl
}

\begin{abstract}
An experimental methodology is proposed for the study of aeroelastic systems. The approach locally evaluates the forces involved in Collar's triangle, namely aerodynamic, elastic, and inertial forces. The position of flow tracers as well as of markers on the object surface is monitored by a volumetric PIV system. From the recorded images, the flow tracers and surfare markers are separated based on their optical characteristics. The resulting images are then analysed by Lagrangian particle tracking. The inertial and elastic forces are obtained solely analysing the motion and the deformation of the solid object, whereas the aerodynamic force distribution is obtained via the pressure-from-PIV technique. Experiments are conducted on a benchmark problem of fluid-structure interaction, featuring a flexible panel installed at the trailing edge of a cylinder. A polynomial fit of the markers' positions is carried out to determine the panel's instantaneous shape, from which the inertial and elastic forces are evaluated. The pressure loads on the panel are determined via solution of the Poisson equation for pressure, imposing adaptive boundary conditions that comply with the panel. The simultaneous measurement of the three forces allows to assess the equilibrium of forces, and in turn to close Collar's triangle.
\end{abstract}

\section{Introduction}

Aeroelastic phenomena occur frequently in nature as well as in a wide number of engineering applications, most notably in civil engineering (Sarkar et al., 1994), energy production (Abdelkefi, 2016, Marshall et al., 1996), and in the transport sector (Wright and Cooper, 2008). In the aeroelastic regime, a flexible body immersed in a flow is subjected to the aeroelastic system of forces, namely aerodynamic, elastic, and inertial. Their mutual relationship was sketched by Collar in 1946 in the so-called Collar's triangle of forces.

Still nowadays, fluid-structure interaction (FSI) problems are difficult to study both from the computational as well as from the experimental viewpoint. From the computational perspective, the one of the main challenges consists of the coupling between the simulation of the fluid flow mathematically with time-varying structural boundaries. Computational aeroelasticity often makes use of simplified geometries (Schuster et al., 2003) to increase affordability. The numerical coupling of independent fluid and structural solvers, while improving the computational efficiency, poses new challenges at the fluidstructure interface, especially for highly non-linear phenomena, such as for unsteady separated flow regimes. This situation justifies the need to advance experimental approaches as a necessary component for the validation of computational tools.

From the experimental perspective, the first challenge is given by the problem of scaling. While Mach and Reynolds numbers are the most relevant parameters for aerodynamic scaling, for FSI problems, one needs to consider the ratio of fluid and structure inertia alongside the relative stiffness of the latter (Friedmann, 2004; Wan and Cesnik, 2014).

Even when the problem is scaled to a satisfactory extent and the experiment is realised, monitoring the fluid flow and structure behaviour is hindered by the technical complexity of the needed instrumentation. Most of the works carrying out simultaneous measurements of flow and structure have been reported only in the last decade, often by combining optical techniques. Many studies have approached the flow measurements by planar particle image velocimetry (Timpe et al., 2013; Hortensius et al., 2017; Kalmbach and Breuer, 2013; Giovannetti et al., 2017; Bleischwitz et al., 2017), and only a few have employed volumetric measurements (Kalmbach and Breuer, 2013; Mitrotta et al., 2019). The 
measurement of the structural motion was performed either by digital image correlation (Timpe et al., 2013; Bleischwitz et al., 2017; Giovannetti et al., 2017) or based on multiple-point laser triangulation (Kalmbach and Breuer, 2013). Moreover, Timpe et al. (2013) directly measured the inertia forces on the structure with a force balance. Furthermore, Mitrotta et al. (2019) used the Lagrangian particle tracking technique (Schanz et al., 2016) to determine simultaneously the flow velocity and the structural motion.

Optical diagnostics for FSI investigation has nowadays advanced to a level that it compares with techniques based on installed instrumentation aboard the structural model. The combined use of PIV techniques (Adrian et al., 2011) and optical diagnostics like digital image correlation or alike (DIC, Chu et al., 1985; Image Pattern Correlation Technique or IPCT, Boden et al., 2014) to perform both tasks is currently under development. Pressure from 3D-PIV enables the evaluation of the fluid flow pressure both in the fluid domain as well as in close proximity of the solid object surface (Liu and Katz, 2006; van Oudheusden, 2013). A pioneering work that applies pressure from PIV to FSI problems with generic three-dimensional and flexible structures has been conducted by Percin et al. (2017).

Finally, the evaluation of inertia forces, traditionally performed by means of accelerometers (as in ground vibration tests, Peeters et al., 2009; or under in-flight conditions, Kehoe, 1995), has also been surrogated by optical diagnostic methods. For example, the videogrammetric model deformation (VMD, Burner et al., 2001) measurement technique captures the attitude of wings by tracking rows of markers on the model. Similarly, a new sensing approach (de Figueiredo et al., 2020) measures natural frequencies of aeronautical structures using a computer vision system.

The present work paves the way towards the closure of Collar's triangle experimentally, that is, determining simultaneously all agents of the dynamic FSI interaction. In order to do so, an experimental methodology is proposed to extract aerodynamic, elastic and inertial forces from LPT measurements of flow tracers and surface markers from a single tomographic system.

\section{FSI measurement working principle}

Let us consider a flexible object, such as a panel of length $L$, width $W$ and thickness $T(T<<L, W)$ subject to aerodynamic forces from an air flow as illustrated in Error! Reference source not found. The aerodynamic loads consist of pressure $p$ and friction $\tau$. The object opposes deformation by its stiffness, and acceleration motions by its inertia. Considering a square tile of such panel, i.e. a small element of side length $l$ (see figure 1), the equilibrium of forces acting on such element reads as:

$$
\sum \boldsymbol{F}=\boldsymbol{F}_{i}+\boldsymbol{F}_{e}+\boldsymbol{F}_{a}=0
$$

where $\boldsymbol{F}_{i}, \boldsymbol{F}_{e}$ and $\boldsymbol{F}_{a}$ stand for the inertia, elastic, and aerodynamic forces, respectively.

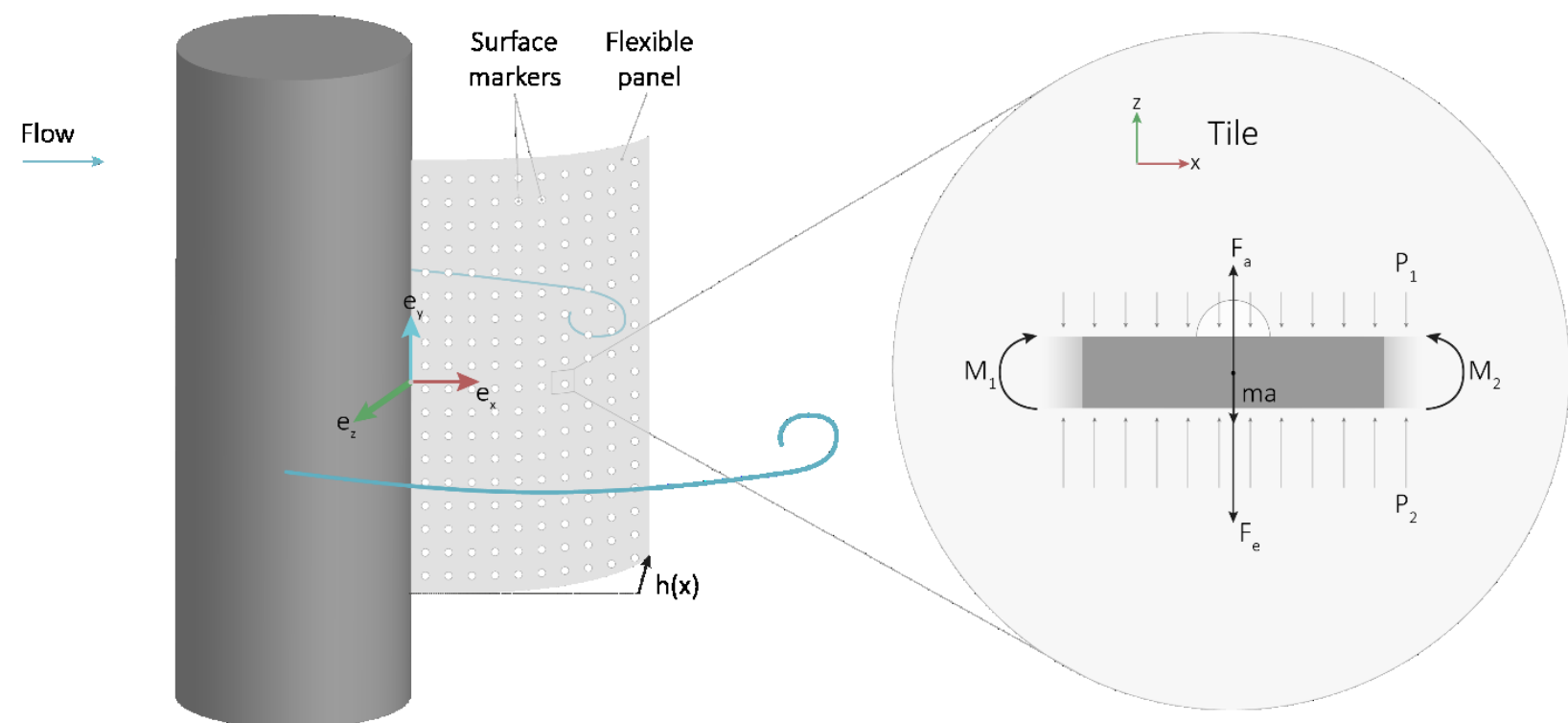

Figure 1: Schematic of a flexible foil immersed in a flow with cross-sectional view of a tile element. 
Inertia force

When the net external forces are non-zero, the tile will accelerate and as such an inertial force $\boldsymbol{F}_{i}$ can be defined in the direction opposite to the acceleration $\boldsymbol{a}$ of the element,

$$
\boldsymbol{F}_{\boldsymbol{i}}=-m \boldsymbol{a}
$$

where $m$ is the tile's mass. The local measurement of the object motion is typically performed by means of optical markers applied onto the surface of the panel. The marker's position in time is recorded, while velocity and acceleration are obtained as time derivatives of the position. The inertial force is thus computed by knowledge of the tile's mass (from the material's density and the tile's volume).

\section{Elastic force}

Elastic forces arise as a reaction of the object opposing deformation. In the present discussion, deformation is assumed to occur in the linear elastic regime. The force is hypothesized to act normal to the surface and tensional forces (e.g. arising from aerodynamic skin friction) are neglected. In the above conditions, the whole elastic force reduces to the bending stress only.

Considering the thinness of the deformable body under study, a 1-D simplification of the KirchhoffLove plate theory provides a relation between the physical attributes of a tile of material and the elastic forces due to a z-deformation $h(x)$, illustrated in figure 1. According to such theory and derived from the compatibility relations (strain-displacement) and the stress-strain relations, the stress along the body reads:

$$
\sigma_{x}=-\frac{E}{1-v^{2}} z\left(\frac{\partial^{2} h}{\partial x^{2}}\right)=\frac{E}{1-v^{2}} z h^{\prime \prime}
$$

where $E$ is the Young modulus and $v$ the Poisson ratio. The linear dependence on $\mathrm{z}$ (with respect to the neutral axis) agrees with the tensile and compressive stresses at opposite faces of the panel. The nonuniform normal stress distribution gives rise to a moment reaction.

Finally, from moment equilibrium for a tile and considering the internal shear forces the normal force acting on the surface and producing such displacement can be inferred:

$$
\boldsymbol{F}_{\boldsymbol{e}}=\frac{E l^{2} T^{3}}{1-v^{2}}\left(\frac{\partial^{4} h}{\partial x^{4}}\right) \boldsymbol{e}_{\mathbf{z}}=\frac{E l^{2} T^{3}}{1-v^{2}} h^{\prime \prime \prime \prime} \boldsymbol{e}_{\mathbf{z}}
$$

The complete derivation of the theory can be found in the work of Panc (1975). Once the material properties are known, the experimental evaluation of such force relies on the assessment of the $4^{\text {th }}$ spatial derivative of the deformation, which in turn could be determined from a dense, yet discrete, description of the deformed foil by a pattern of surface markers.

\section{Aerodynamics force}

Aerodynamics loads are usually decomposed into normal (pressure) and shear (friction) stresses. Friction is negligible in most aeroelastic regimes. Moreover, the pressure loads on a thin panel reduce the discussion to the pressure difference $\Delta p$ at its opposite sides. The aerodynamic force acting on the tile reads as:

$$
\boldsymbol{F}_{\boldsymbol{a}}=\Delta p l^{2} \boldsymbol{n}
$$

where $\boldsymbol{n}$ is unit vector in the normal direction to the surface. The pressure field can be determined from the velocity field information invoking the momentum equation, in turn requiring the solution of the Poisson equation for pressure (van Oudheusden, 2013). Such force is less straightforward to obtain from fluid flow measurements as it typically requires high-quality time-resolved 3D measurements (Beresh, 2021). Moreover, the choice of boundary conditions can largely affect the evaluation of the pressure field (Neeteson et al., 2016). Furthermore, the surface pressure is typically estimated extrapolating the pressure from the flow domain towards the solid surface (Jux et al., 2020). It should be stressed that for evaluating the surface pressure distribution over a generic surface, curved and in motion, time-resolved three-dimensional flow field measurements are required that surround such surface.

Collar's equilibrium of forces

Equation 1 can be rewritten combining the explicit terms obtained in eq. 2, 4 and 5. The resulting equilibrium of forces is expressed as: 


$$
\frac{E l^{2} T^{3}}{1-v^{2}} h^{\prime \prime \prime \prime} \boldsymbol{e}_{\mathbf{z}}+\Delta p l^{2} \boldsymbol{n}-m \boldsymbol{a}=0
$$

From such equation, we can identify some describing parameters of the structural element considered: size $(l, T)$, mass $(m)$ and material properties (Young modulus, $E$, and Poisson ratio, $v$ ). Instead, the remaining elements in the equation, such as the pressure difference $\Delta p$, the element acceleration $\boldsymbol{a}$, orientation $\boldsymbol{n}$, and derivatives of the deformation $h^{\prime \prime \prime \prime}$, must be measured to address the problem.

In the present study, state-of-the-art optical diagnostics based on PIV techniques is employed for the above purpose. The fluid flow velocity is obtained over an extended three-dimensional domain by tracking large tracers (helium-filled soap bubbles, HFSB) along their trajectories. This data is used to ultimately determine the flow pressure in the vicinity of the body. The acceleration of the panel is measured by tracking the motion of surface markers with the same algorithm as for the fluid tracers. Finally, the orientation and the deformation of the panel can be inferred from the analysis of the 3D position of the markers and their relative displacement, respectively. Section 3 details the complete description of the experimental approach to measure these unknowns $\left(\Delta p, \boldsymbol{a}, \boldsymbol{n}, h^{\prime \prime \prime \prime}\right)$ with such methodologies.

\section{Experimental setup}

\section{Flow facility and model}

The experiments are conducted at the Aerospace Engineering laboratories of TU Delft, with an open-jet open-return wind-tunnel of $60 \times 60 \mathrm{~cm}^{2}$ exit cross-section. A black solid cylinder $(D=90 \mathrm{~mm})$ is located vertically with a flexible transparent foil attached along the rear mid-plane. The see-through characteristic of the foil allows to track flow tracers at both sides of the body even when looking from only one side. The foil size $\left(105 \times 200 \mathrm{~mm}^{2}\right)$ is such that the foil undergoes a 2D motion following the Karman wake of the cylinder at $U_{\infty}=2.25 \mathrm{~m} / \mathrm{s}\left(R e=1.35 \times 10^{4}\right)$.

For the measurement of the foil kinematics, a regular pattern of bright surface markers (see Fig. 2C) is speckled on the foil by hand. The markers had an average diameter $d_{m}=0.8 \mathrm{~mm}$ and a pitch $p_{m}=1 \mathrm{~cm}$. The overall experimental setup is provided in figure $2 \mathrm{~A}$.

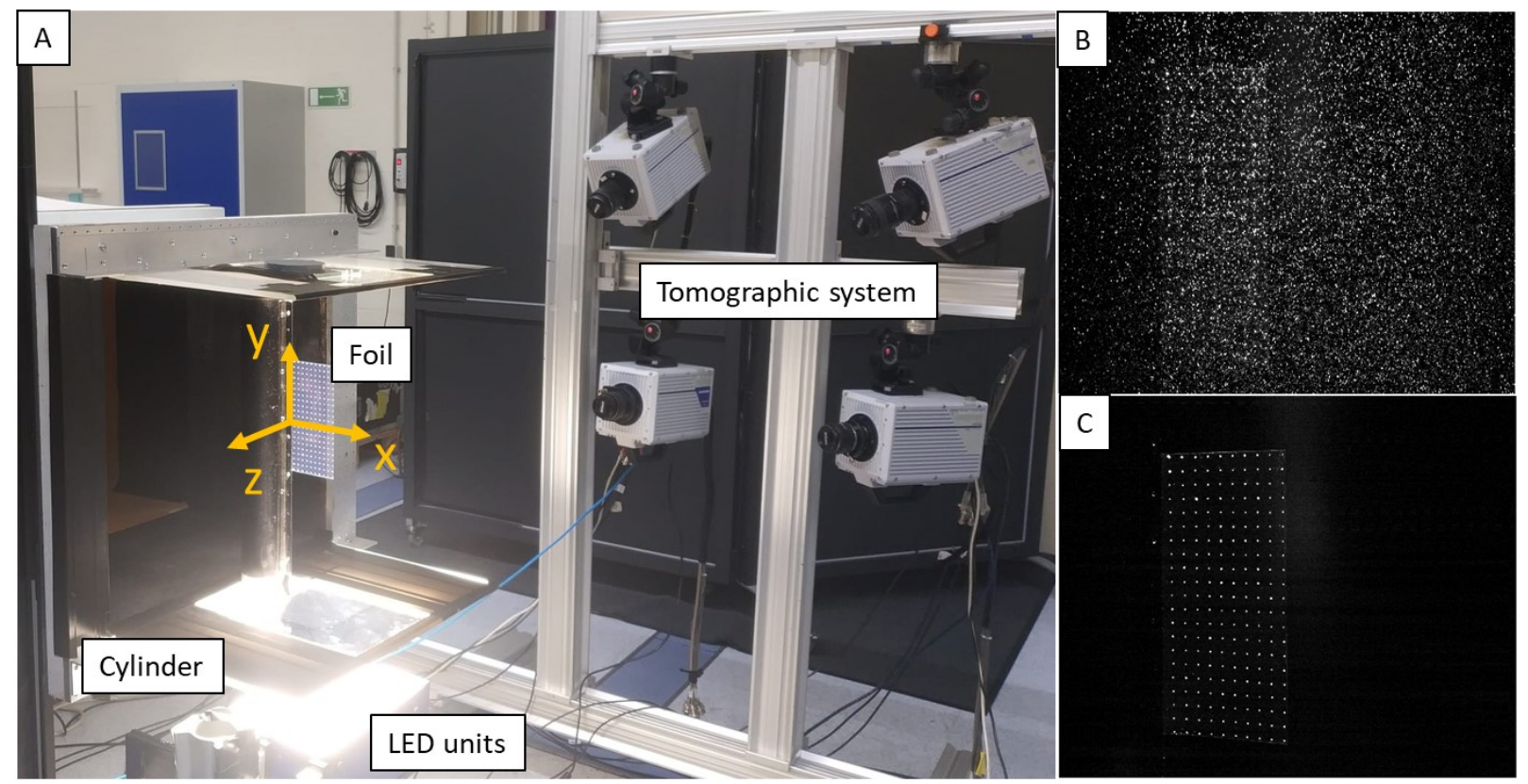

Figure 2: Experimental setup (A) showing the model (foil attached to a cylinder), the LED units. Raw snapshots of foil markers with HFSB (B) and without (C).

Table 1: Physical properties of the flexible foil and tile

$\begin{array}{ll}\text { Thickness, } T[\mathrm{~mm}] & 0.25 \\ \text { Length, L }[\mathrm{mm}], 1[\mathrm{~mm}] & 95,10\end{array}$




\begin{tabular}{ll} 
Width, W [mm $], w=1[\mathrm{~mm}]$ & 200,10 \\
Density of material, $\rho\left[\mathrm{kg} / \mathrm{m}^{3}\right]$ & 1290 \\
Young modulus of material, E [GPa] & 1.0 \\
Poisson ratio of material, $v[-]$ & 0.3 \\
\hline
\end{tabular}

Tracers measurement

The flow is seeded with neutrally buoyant sub-millimetre $(d \approx 350 \mu m)$ helium-filled soap bubbles (HFSB, Scarano et al., 2015), released by an in-house developed 200-generator seeding rake installed in the settling chamber. The nominal production rate of flow tracers is $6 \times 10^{6}$ bubbles/s distributed over a section of $120 \times 50 \mathrm{~cm}^{2}$, resulting in a seeding concentration of $\sim 10 \mathrm{bubbles} / \mathrm{cm}^{3}$ for a freestream velocity $U_{\infty}=2.25 \mathrm{~m} / \mathrm{s}$ at the test section. For the current experiment, only half seeder was operated to keep the seeding concentration below the optical limit of 0.05 particles per pixel $(p p p)$ for 4-camera tomographic systems (Elsinga et al, 2006; Scarano, 2013).

A seeded volume of $22 \times 40 \times 20 \mathrm{~cm}^{3}$ is illuminated vertically by two LaVision Flashlight 300 LED banks located under the test section. The imaging system consisted of four high-speed cameras (1024x1024 pixels,12-bit, $20 \square \mathrm{m}$ pixel pitch) mounted in a tomographic configuration as shown in figure 2 . The cameras subtended an aperture of approximately 30 degrees.

The image acquisition and processing is conducted with the LaVision Davis 10.0.5 software. Sets of 5000 images are recorded at a rate of $1 \mathrm{kHz}$. For the calibration process, a geometrical calibration (Soloff et al. 1997) is followed by the volume self-calibration (Wieneke 2008) and optical transfer function determination (OTF calibration, Schanz et al 2012). Surface markers are visually distinguished from flow tracers based on their distinct optical characteristics. In order to track the most fluid tracers (3D bubbles) and the most structural markers (2D flat bright circles), the volume self-calibration and the OTF calibration are performed independently with a clean run of each tracers. The flow tracers' velocity is evaluated by means of the Shake-the-Box algorithm (Schanz et al., 2016) to the overall measurement domain.

As for the flow tracers, the motion of the markers is determined by means of the STB processing. The analysis volume is reduced to the bounded moving foil to avoid retrieving flow tracers. In addition, given the periodic $2 \mathrm{D}$ nature of the foil motion, velocity limits within the tracking algorithm aid to discard flow particles with a stream-wise motion.

Table 2: Optical system information.

\begin{tabular}{|c|c|}
\hline \multicolumn{2}{|l|}{ Tomographic system } \\
\hline Illumination & 2 x LaVision Flashlight 300 LED \\
\hline Cameras & $\begin{array}{l}2 \times \text { Photron FastCam SA1.1 (1024x1024 pixels, } 5400 \mathrm{fps}) \\
1 \times \text { Photron FastCam SA5 }(1024 \times 1024 \text { pixels, } 7000 \mathrm{fps}) \\
1 \text { x HighSpeedStar } 8(1024 \times 1024 \text { pixels, } 7000 \mathrm{fps})\end{array}$ \\
\hline Imaging & $\begin{array}{l}3 \times \text { Objectives Nikkor } 50 \mathrm{~mm}-\mathrm{f} \# 1 / 11 \\
1 \text { x Objectives Nikkor } 60 \mathrm{~mm}-\mathrm{f \#} 1 / 11\end{array}$ \\
\hline Acquisition frequency & $1000 \mathrm{~Hz}$ \\
\hline
\end{tabular}

\section{Data reduction for load estimation}

The following section describes the data reduction procedure to obtain the distribution of forces along the foil from the analysis of the tracers and markers motion.

\section{$\underline{\text { Structural forces }}$}

As introduced in section 2, inertial and elastic forces are determined via the computation time and space derivatives of the measured foil location. In order to avoid noise propagation through the derivation process, a spatio-temporal regularization is performed on the locations of the markers to fit a coherent surface. The fit is built upon a $5^{\text {th }}$ order polynomial in space that comprises a time stencil of 17 samples ( $\sim 10 \%$ of a cycle). The function is calculated on a finer grid with $0.5 \mathrm{~cm}$ spacing in $\mathrm{x} \& \mathrm{y}$ directions. 
Therefore, the resulting tiles have a surface area of $0.25 \mathrm{~cm}^{2}$. The derivations in time and space to determine the acceleration and elastic force, respectively, are conducted analytically based on the polynomial description of the foil's instantaneous shape.

Figure 3 compares the kinematics in time of a tracked surface marker with the kinematics of its projection on the reconstructed surface. Figure 3-right illustrates a clear suppression of the noise in the foil's acceleration.
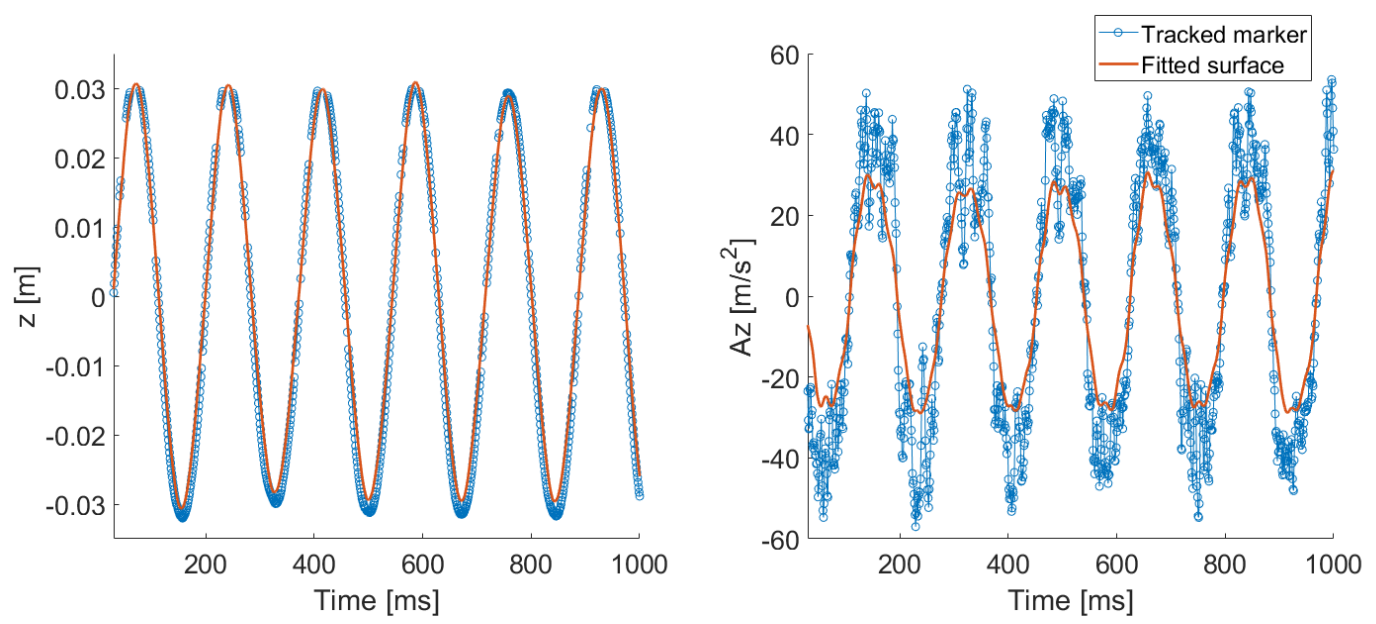

Figure 3: Kinematics of a measured surface marker (dotted blue) and projection at fitted surface (solid

Fluid load red) at the location $(x=0.75 \mathrm{~L}, \mathrm{y}=0.5 \mathrm{~W})$.

Finally, for the determination of the aerodynamic forces, the surface pressure distribution along the foil needs to be evaluated. The volumetric pressure is computed based on the solution of the Poisson equation for pressure for incompressible flows (van Oudheusden, 2013), which results from the divergence of the equation of conservation of momentum:

$$
\nabla^{2} p=\nabla \cdot\left(-\rho \frac{D \bar{u}}{D t}+\mu \nabla^{2} \bar{u}\right)
$$

The computation of the pressure field relies on the material derivatives of the velocity, $\frac{D \bar{u}}{D t}$, and the viscous dissipation, $\mu \nabla^{2} \bar{u}$. The latter is neglected due to the high flow Reynolds number $(\operatorname{Re}=2 \times$ $10^{4}$ ). The material derivatives are directly extracted from the PTV measurements, in which particles are tracked along their trajectories in a Lagrangian fashion.

Dense velocity reconstruction from particle tracks is performed with the VIC\# algorithm implemented in DaVis 10.1 (Jeon et al., 2018). The approach is based on the Vortex-in-Cell method, which optimizes the vorticity field to best fit the velocity tracks via an optimization algorithm that minimizes a cost function $\mathrm{J}$ built upon experimental-computational variables disparities. Pressure integration is performed simultaneously to the velocity reconstruction since the pressure is computed within the optimization algorithm and it is included in the cost function of VIC\# (Jeon et al, 2018). Pressure fields are computed by solving the Poisson equation with Neumann conditions at every boundary and a constant Dirichlet condition at a free stream location.

Similarly to most FSI applications, the current case is characterized by unsteady fluid-solid boundaries. However, the application of the VIC\# methodology limits its applicability to Cartesian domains, limiting the knowledge of the pressure field from the outer-most region of the measurement domain down to the limits of the foil motion ( $4 \mathrm{~cm}$ away from the neutral position of the unperturbed foil). The gap is such that the foil does not enter the fluid domain under consideration. Thus, a Cartesian mesh of fluid domain outside the range of motion of the foil is selected for fine-gridding the velocity field and computing the flow static pressure field. For the determination of the actual surface pressure, the pressure is extrapolated towards the foil location (from both sides) via a nearest neighbour approach, neglecting the change of static pressure across the wake. 


\section{Results}

The flow velocity fields are analysed first. A full field $\mathrm{x}-\mathrm{z}$ view of the velocity vectors is captured in figure 4, averaged along the y-direction. The foil location is given as a reference. The spanwise component of the velocity does not provide relevant information and so it is omitted. The fields show the wake of the cylinder as a low velocity region. From the streamwise component (top row in Fig. 4), the wake undergoes an oscillatory behaviour with deflections of $\pm 20^{\circ}$ with respect to the $x$-axis. The vortex shedding phenomenon is also reflected in the transverse velocity component, which shows positive and negative velocity regions coincident with the upwards-downwards wake deflections respectively.

The dominant frequency of the flow can be already inferred from the sequence, $f \approx 5.6 \mathrm{~Hz}$. The latter agrees with the characteristic frequency for cylinder shedding wakes, $S t \approx 0.2$ (Williamson, 1996), showing no effect the existence of the flexible foil at the trailing edge of the cylinder.
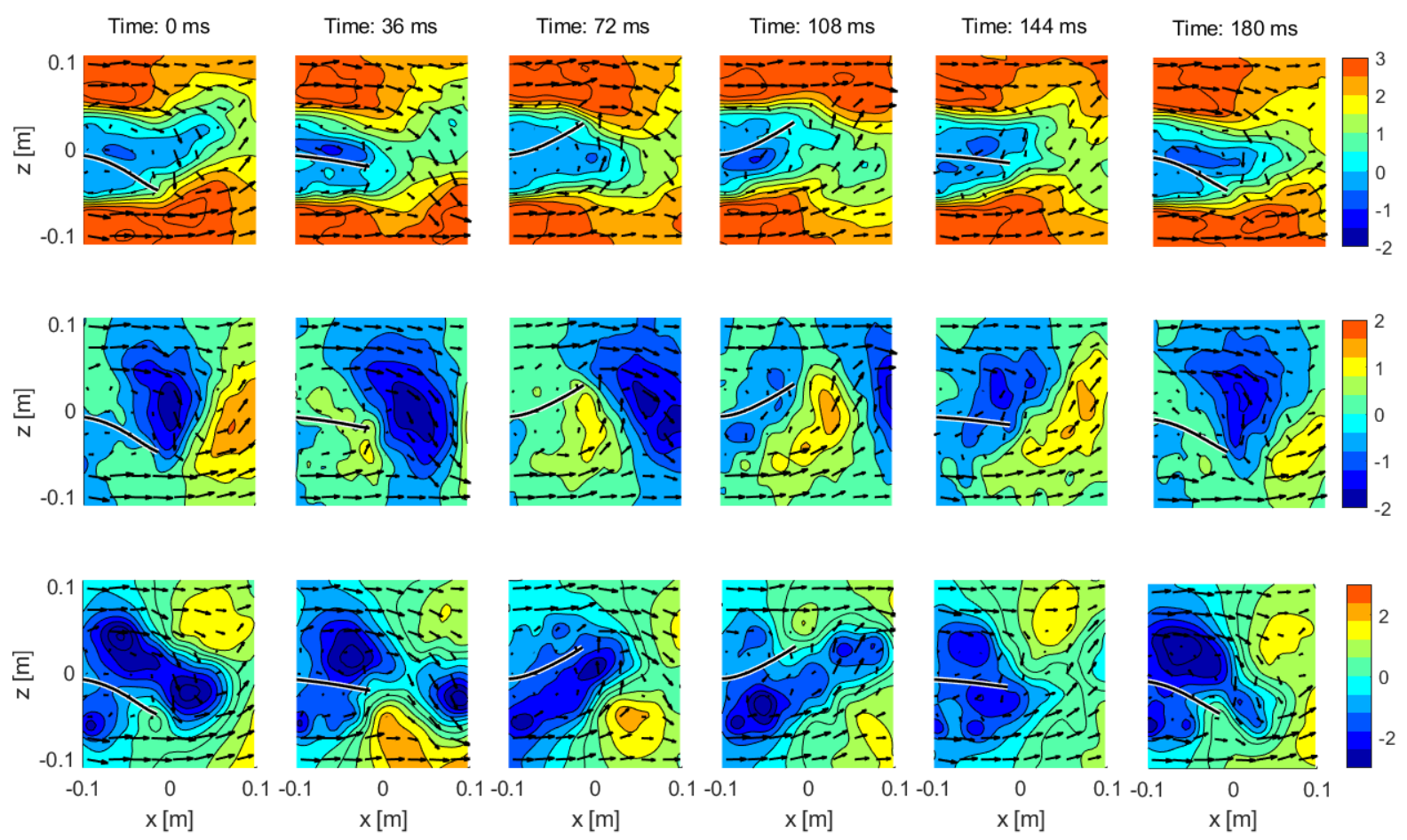

Figure 4: Sequence (left to right) of average (along the y-direction) flow velocity fields ( $u$ and w, top and mid rows respectively in $[\mathrm{m} / \mathrm{s}]$ ) and pressure field (bottom row in $[\mathrm{Pa}]$ ).

The pressure fields, which are provided in the third column of Fig. 4, give an insight of the forcing mechanism of the problem. Focusing on the low pressure regions (dark blue), it is observed how the latter pull on the foil creating the oscillatory motion. In particular, the actual forcing appears to happen when the low pressure region convects from the foil mid-length $(x=0.5 \mathrm{~L})$ to the end of the foil $(x=\mathrm{L})$. In this situation, both pulling force and velocity vector are aligned, and translates into a positive work performed by the flow on the foil. This mechanism is enhanced by the high pressure regions (redorange), which build up at the outer region when the foil is at the limit of the motion (when the foil is still) and aid to push the foil back within the oscillatory fashion.

About the structural motion, the complete 4D description of the foil is provided in figure 5 . Contours of position, acceleration and elastic force for the spanwise direction (z-) are provided on the deformed state of the foil. The acceleration of the foil (centre column) follows the inverse trend to the motion itself, as expected from the second derivative a given periodic signal. Similarly, the elastic forces oppose to the foil position, as the deformation promotes internal stresses and moments that act against it. This working mechanism is analogous to that of a mass-spring system under deformation. 

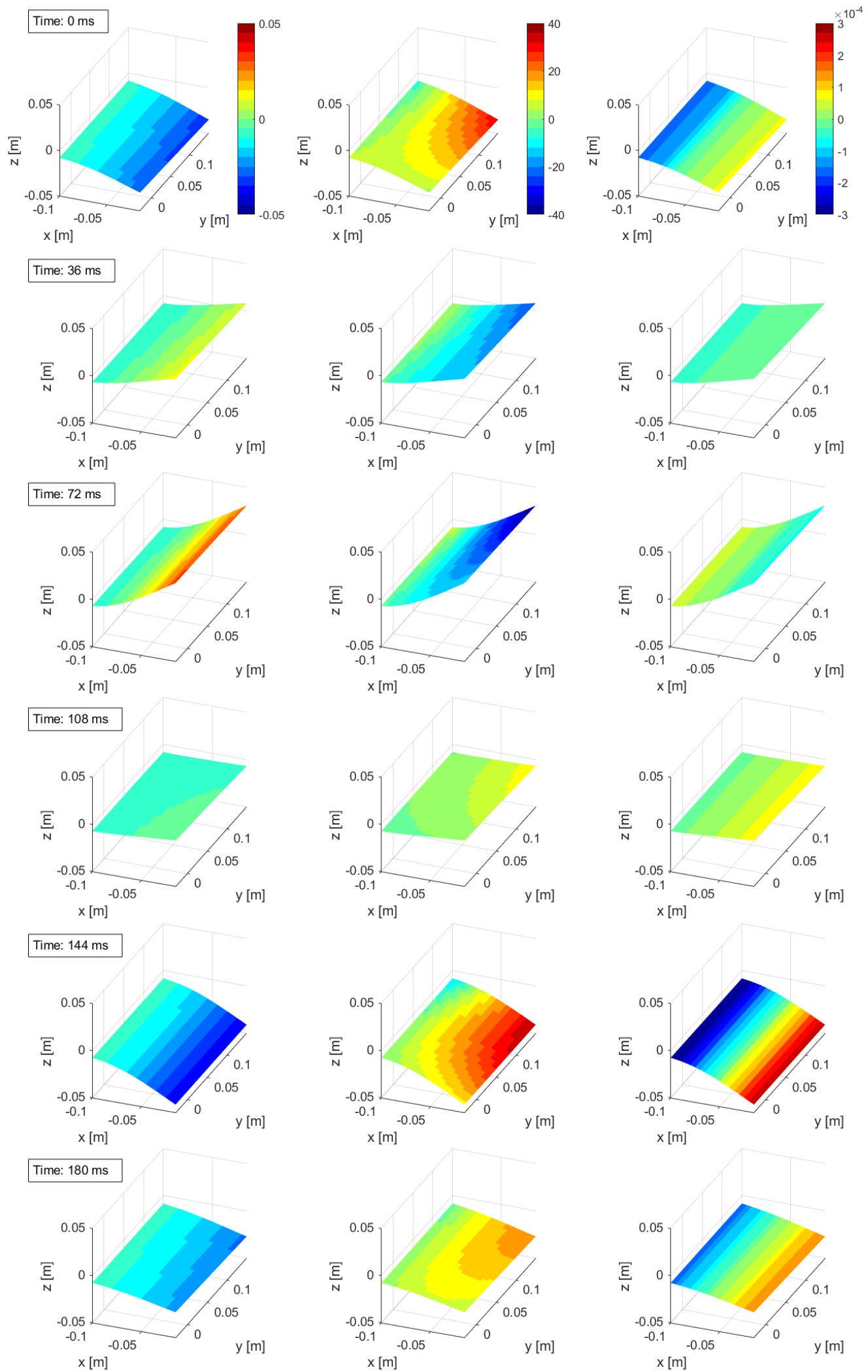

Figure 5: Sequence (top to bottom) of structural variables contours on the deformed foil: z-location (left column, in $[\mathrm{m}]$ ), $\mathrm{z}$ - acceleration (related to the inertia forces, centre column, in $\left[\mathrm{m} / \mathrm{s}^{2}\right]$ ), and zelastic force (right column, in $\left[\mathrm{N} / \mathrm{cm}^{2}\right]$ ).

The time-evolution of the computed aeroelastic forces is extracted at a particular tile location $(\mathrm{x}=0.75 \mathrm{~L}, \mathrm{y}=0.5 \mathrm{~W})$ and presented in figure 6 . The $\mathrm{z}$-position of such tile is included as a reference as a purple dashed line. A first reading can be done on the relative phase of the forces with respect to the 
motion of the foil. It can be seen that the tile's inertia is in-phase with its z-displacement. This result can be explained via the following reasoning. Considering the z-position as a pseudo-sinusoidal signal, the second time derivative (tile acceleration) results in a counter-phase signal, i.e. a signal with $\pi$ phaseshift. However, recalling eq. 2, the inertia (red line in fig. 6) has opposite sign to the acceleration, resulting in an in-phase signal with the foil deformation (dashed black in fig. 6). In fact, the inertia acts to maintain the motion of the foil, opposing to accelerations caused by other external forces. In contrast, the elastic forces (yellow in fig. 6) oppose the deformation, reacting to bring the foil back to the nondeformed state. Therefore, the elastic forces are in counter-phase with the z-position. However, as indicated in the previous section, the computation of the latter forces relies on the $4^{\text {th }}$ spatial derivative of the deformation, which causes measurement noise to affect the accurate evaluation of the elastic forces. Last but not least, the aerodynamic force (blue in fig. 6) shows lower force levels and anticipates the foil's motion by a phase shift of about $\pi / 2$. This phase shift is caused by the structural and aerodynamic damping of the system . In order to perform work, and so induce the motion to the foil, the external force, i.e. aerodynamic pressure difference across the body, must align with the velocity of the body. The velocity and the position present a $\pi / 2$ phase shift, thus a similar phase-shift occurs between the aerodynamic force and the z-location of the foil.

Finally, the closure of Collar's triangle, i.e. force equilibrium residual (eq. 1), is evaluated for the afore-analysed tile location $(\mathrm{x}=0.75 \mathrm{~L}, \mathrm{y}=0.5 \mathrm{~W})$ and provided in fig. 6 as black dash-dotted line. A priori, one could expect the residual to be incoherent in time and dominated by random noise. However, the signal presents the dominant frequency of the motion in-phase to the inertial forces. This behaviour possibly indicates an underestimation of the elastic and/or aerodynamic loads on the foil.

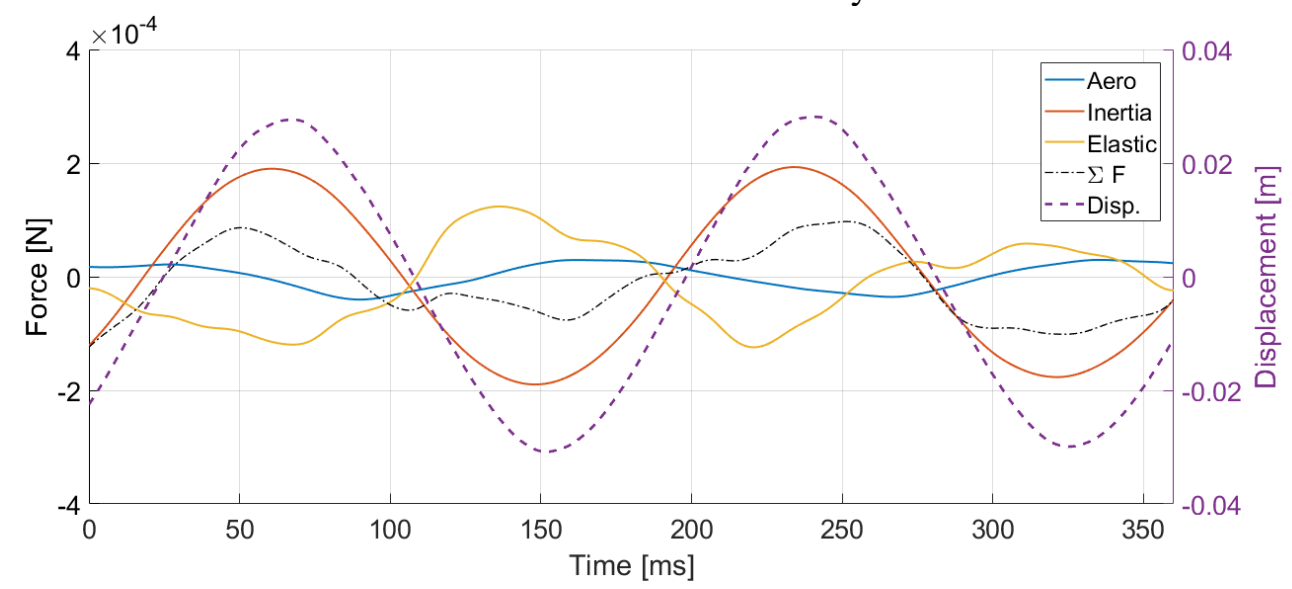

Figure 6: Instantaneous time-evolution of aeroelastic forces: aerodynamic (blue), inertial (red), and elastic (yellow) for the location $(\mathrm{x}=0.75 \mathrm{~L}, \mathrm{y}=0.5 \mathrm{~W})$. The sum of forces is drawn in black dash-dotted. $\mathrm{Z}$-position (purple dashed) is given as reference (right y-axis).

\section{Conclusions}

A non-intrusive measurement methodology for aeroelastic experiments based on the Lagrangian particle tracking technique is proposed to simultaneously estimate aerodynamic, elastic and inertial loads from a deforming model. The method relies on the tracking of flow tracers to characterize the flow, and of surface markers to describe the dynamics of the structure. An experiment was carried out to show the applicability of the proposed methodology. The measurements regarded a flexible transparent foil, attached to a solid cylinder, interacting with the wake of the cylinder. Both flow tracers, HFSB, and surface markers, installed on the panel, were tracked in time with a single optical tomographic system.

The results show the potential of measuring the surface markers position, velocity and acceleration via a tracking approach for load estimation. Structural forces where successfully estimated on the flexible foil. For the current work, aerodynamic pressure on the surface of the moving body was extrapolated from the Cartesian fluid domain via the nearest neighbour approach. A challenge to overcome in future works is the surface determination of the unsteady pressure for a deforming body in motion. Nevertheless, the behaviour of the computed forces time-evolution are consistent with the hypothesized trends. Yet, the noise propagation through the spatial derivation process affects the accuracy of the estimated elastic forces, precluding the closure of the aeroelastic triangle. 
To conclude, the proposed methodology offers a wide spectrum of information (flow fields, flow topology, fluid loads, structural deformations, and structural loads) based on non-intrusive optical measurements by Lagrangian Particle Tracking. Moreover, the simultaneous estimation of the main forces involved in an aeroelastic experiment implies the ability to close Collar's triangle, bringing promising advantages to the experimental study of the challenging aeroelastic regimes.

\section{References}

Abdelkefi A (2016) Aeroelastic energy harvesting: A review. International Journal of Engineering Science 100:112-135

Adrian L, Adrian RJ and Westerweel J ( 2011) Particle image velocimetry. Cambridge university press.

Beresh S (2021) Time-resolved particle image velocimetry. Measurement Science and Technology

Bleischwitz R, De Kat R and Ganapathisubramani B (2017) On the fluid-structure interaction of flexible membrane wings for MAVs in and out of ground-effect. Journal of Fluids and Structures 70:214-234

Boden F, Kirmse T, Poroikov AY, Rinkevichyus BS, Skornyakova NM and Shashkova IA (2014) Accuracy of measurement of dynamic surface deformations by the image pattern correlation technique. Optoelectronics, instrumentation and data processing 50(5):474-481

Burner AW and Liu T (2001) Videogrammetric model deformation measurement technique. Journal of Aircraft 38(4):745-754

Chu TC, Ranson WF and Sutton MA (1985) Applications of digital-image-correlation techniques to experimental mechanics. Experimental mechanics 25(3):232-244

Collar AR (1946) The expanding domain of aeroelasticity. The Aeronautical Journal 50(428):613-36

De Figueiredo HV, Castillo-Zúñiga DF, Costa NC, Saotome O and da Silva RGA (2020) Aeroelastic Vibration Measurement Based on Laser and Computer Vision Technique. Experimental Techniques, 1-13.

Elsinga GE, Scarano F, Wieneke B and van Oudheusden BW (2006) Tomographic particle image velocimetry. Experiments in fluids 41(6):933-947

Friedmann PP (2004) Aeroelastic scaling for rotary-wing aircraft with applications. Journal of fluids and structures 19(5):635-650

Giovannetti LM, Banks J, Turnock SR and Boyd SW (2017) Uncertainty assessment of coupled Digital Image Correlation and Particle Image Velocimetry for fluid-structure interaction wind tunnel experiments. Journal of Fluids and Structures 68:125-140

Hortensius R, Dutton JC and Elliott GS (2017) Simultaneous planar PIV and sDIC measurements of an axisymmetric jet flowing across a compliant surface. In 55th AIAA Aerospace Sciences Meeting (p. 1886).

Jeon YJ, Müller M, Michaelis D and Wieneke B (2018) Efficient reconstruction of flow field with pressure from particle tracks: VIC. Bulletin of the American Physical Society 63

Jux C, Sciacchitano A, Scarano F (2020) Flow pressure evaluation on generic surfaces by robotic volumetric PTV. Measurement Science and Technology 31(10):104001.

Kalmbach A and Breuer M (2013) Experimental PIV/V3V measurements of vortex-induced fluid-structure interaction in turbulent flow-A new benchmark FSI-PfS-2a. Journal of Fluids and Structures 42:369-387

Kehoe MW (1995) A historical overview of flight flutter testing.

Liu X and Katz J (2006) Instantaneous pressure and material acceleration measurements using a four-exposure PIV system. Experiments in fluids 41(2):227

Panc V (1975) Theories of elastic plates. Springer Science \& Business Media.

Peeters B, Hendricx W, Debille J and Climent H (2009) Modern solutions for ground vibration testing of large aircraft. Sound and vibration 43(1):8

Percin M, Vanierschot M and Van Oudheusden BW (2017) Analysis of the pressure fields in a swirling annular jet flow. Experiments in fluids 58(12):166

Marshall JG and Imregun M (1996) A review of aeroelasticity methods with emphasis on turbomachinery applications. Journal of fluids and structures 10(3):237-267

Mitrotta FM, Sciacchitano A, Sodja J, De R. and Breuker BW (2019) Experimental investigation of the fluidstructure interaction between a flexible plate and a periodic gust by means of Robotic Volumetric PIV. In Proceedings of the 13th International Symposium on Particle Image Velocimetry. Universitat der Bundeswehr Munchen.

Neeteson NJ, Bhattacharya S, Rival DE, Michaelis D, Schanz D, Schröder A (2016) Pressure-field extraction from Lagrangian flow measurements: first experiences with 4D-PTV data. Experiments in Fluids 57(6):1-8.

Timpe A, Zhang Z, Hubner J and Ukeiley L (2013) Passive flow control by membrane wings for aerodynamic benefit. Experiments in fluids 54(3):1471

Sarkar PP, Jones NP and Scanlan RH (1994) Identification of aeroelastic parameters of flexible bridges. Journal of Engineering Mechanics 120(8):1718-1742 
Scarano F, Ghaemi S, Caridi GCA, Bosbach J, Dierksheide U and Sciacchitano A (2015) On the use of heliumfilled soap bubbles for large-scale tomographic PIV in wind tunnel experiments. Experiments in Fluids 56(2):42

Schanz D, Gesemann S and Schröder A (2016) Shake-The-Box: Lagrangian particle tracking at high particle image densities. Experiments in fluids 57(5):70

Schanz D, Gesemann S, Schröder A, Wieneke B and Novara M (2012) Non-uniform optical transfer functions in particle imaging: calibration and application to tomographic reconstruction. Measurement Science and Technology 24(2):024009

Schuster DM, Liu DD and Huttsell LJ (2003) Computational aeroelasticity: success, progress, challenge. Journal of Aircraft 40(5):843-856

Soloff SM, Adrian RJ and Liu ZC (1997) Distortion compensation for generalized stereoscopic particle image velocimetry. Measurement science and technology, 8(12), p.1441.

Van Oudheusden BW (2013) PIV-based pressure measurement. Measurement Science and Technology 24(3):032001

Wan Z and Cesnik CE (2014) Geometrically nonlinear aeroelastic scaling for very flexible aircraft. AIAA Journal 52(10):2251-2260

Wieneke B (2008) Volume self-calibration for 3D particle image velocimetry. Experiments in fluids 45(4):549556

Williamson CH (1996) Vortex dynamics in the cylinder wake. Annual review of fluid mechanics 28(1):477-539

Wright JR and Cooper JE (2008) Introduction to aircraft aeroelasticity and loads. John Wiley \& Sons. 